\title{
Prevalence, Distribution, Risk factors and Antifungal Susceptibility Profiles of Candida species in a Tertiary Care Hospital
}

\author{
Sonu Panwar ${ }^{1 *}$ and Sameer Singh Faujdar ${ }^{2}$ \\ ${ }^{1}$ School of Medical Sciences and Research, Grater Noida, Uttar Pradesh, India \\ ${ }^{2}$ Santosh Medical College and Hospital, Ghaziabad NCR Delhi, India \\ *Corresponding author
}

\section{Keywords \\ Candida species, Candidiasis, Vulvovaginitis, Antifungal Susceptibility, C. tropicalis.}

\begin{tabular}{l}
\hline Article Info \\
\hline Accepted: \\
15 March 2016 \\
Available Online: \\
10 April 2016
\end{tabular}

A B S T R A C T
Candidiasis has emerged as an alarming opportunistic disease with the increase in number of patients who are immunocompromised, aged, receiving prolonged antibacterial and aggressive cancer chemotherapy or undergoing invasive surgical procedures and organ transplantation. In last few decades, there have been numerous reports of Candida infections in India. Candida albicans is considered to be the commonest and most virulent pathogenic species of the genus Candida. The clinical specimens investigated included cervical swab constituting 41(34.17\%), skin scrapings 18(15\%), oral swabs 14(11.67\%), nail scrapings $12(10 \%)$, ear swabs $11(9.17 \%)$, sputum $10(8.33 \%)$ followed by pus $7(5.83 \%)$, urine $3(2.50 \%)$, blood $2(1.67 \%)$ and stool $2(1.67 \%)$. These samples were collected from Sharda Medical College \& Hospital a tertiary care hospital. Candida species were identified from clinical samples by microscopy (KOH, Wet mount), culture on SDA and urease test. Further the isolates are speciated by germ tube test, CMA morphology, CHROM agar, sugar assimilation and sugar fermentation tests. Antifungal susceptibility was done by Disc diffusion method. Candidiasis was found to be occurring in all ages but predominant in 20-40 age group with slight increase in extreme ages. Females constituted $55 \%$ with ratio of male to female $1: 1.22$. The most common form of candidiasis found to be Candidial vulvovaginitis (34\%) followed by intertrigo (15\%). Pregnancy $(21.67 \%)$ and HIV infection (20.83) appeared to be the major predisposing factor followed by diabetes (15.83\%). Candida albicans $(74.17 \%)$ found to be the major species isolated followed by non albicans candida $(25.87 \%)$ mainly C. tropicalis $(17.5 \%)$, C. glabrata $(6.67 \%)$ and C. krusei $(1.67 \%)$. Antifungal susceptibility testing results showed the resistance to commonly used antifungal agents in varying proportions in all types of species isolated. Resistance for Fluconazole was 15.83\%, Ketoconazole 10\%, Nystatin 4.17\% and Amphotericin B 1.67\%. Disc diffusion method is simple reliable and easy to perform and should be suitably standardized in the laboratory.

\section{Introduction}

Candida species are the most common causes of fungal infection. Candida is normal inhabitant in the skin, mucous membrane of oral cavity including gastrointestinal tract, respiratory tract and genitourinary tract and may invade other parts of the body especially in immunocompromised individuals (Rippon, 1998). Candidiasis is mainly caused by Candida albicans, while there has been 
striking increase in the frequency with non albicans Candida species in last few years.

The most important species which are considered pathogenic to human are C.albicans, C. tropicalis, C. kruseii, C.glabrata, C.lusitaniae and C.viswanathii (Chander Jagadish 2002). Predisposing factors for candida infection are: prolonged use of antimicrobial agents, immunocompromised status, chemotherapy, catheterization, aging, pregnancy, diabetes and secondary to bacterial infections (Cheryl M, Morrison CJ 1998). Candidiasis is usually endogenous in origin. The spectrum of disease caused by candida is extensive. The range of manifestations extends from simple mucosal colonization to multiple organ invasion or invasive candidiasis in the neonate and elderly. Candida can also cause nosocomial infections (Alan. M. Sugar et al., 1997).The Candida species are the 4th most common organisms causing blood stream infection, and constitute $8 \%$ of all nosocomial infections. Candida infections are the second most frequently diagnosed opportunistic infections in patients with human immunodeficiency virus. Also three of every four women will have at least one bout of vulvovaginal candidiasis during lifetime (Jarwis, 1995 \& Murray MP et al., 2005).

Patients admitted at tertiary care hospitals have access to very intensive management modalities. Due to variable clinical presentation of candida infections, it becomes very important to identify this pathogens from all theclinical specimens received at laboratory irrespective of clinician's suspicion. Candida species differ in their antifungal susceptibility and virulence factors. Thus identification of candida up to species level along with antifungal susceptibility becomes very essential (Jawetz et al., 1978\&V.
Manchanda et al., 2011). The objectives of this study were to identify potential risk factors associated with Candida infections and their distribution in various clinical specimens along with antifungal susceptibility status.

\section{Materials and Methods}

The present study was conducted for a period of one year from March 2014 to February 2015 at the School of Medical Sciences and Research, Grater Noida, Uttar Pradesh, India. All the clinical specimens (oral swabs, ear swabs, vaginal swabs, stool, CSF, sputum, blood, pus, nail scrapings etc.) submitted to the microbiology laboratory suspected of fungal infection during the study period constituted the material. 120 specimens met the study inclusion criteria which were further evaluated, demographic and clinical data such as age, sex, site of infection, predisposing factors, history of exposure to antifungals and clinical outcomes of patients were noted. Candida isolates were screened for gram staining, $20 \%$ or $40 \% \mathrm{KOH}$ (Potassium Hydroxide) mount, culture characteristics on Sabouraud's dextrose agar (SDA) with Chloramphenicol $(50 \mathrm{mg} / \mathrm{L})$ and gentamycin (20 $\mathrm{mg} / \mathrm{L})$ and urease test. The candida isolates which were obtained further speciated by the germ tube test, chlamydospore formation on cornmeal agar (by doing Dalmau technique), inoculation on chromogenic medium (HiMedia CHROM agar), sugar fermentation and sugar assimilation test. Anti-fungal susceptibility was performed by disc diffusion method using Yeast nitrogen base agar. Discs used were Amphotericin B $(10 \mu \mathrm{g})$, Fluconazole $(10 \mu \mathrm{g}), \quad$ Nystatin $\quad(10 \mu \mathrm{g})$, Ketoconazole $(10 \mu \mathrm{g})$ and to determine whether the isolates tested against amphotericin B, nystatin, fluconazole, ketoconazole were susceptible, intermediate 
or resistant; the diameters of zones of inhibition obtained were compared with the standard Zones interpretive break points published by CLSI M44-A215 guidelines.

\section{Results and Discussion}

Total 120 isolates of Candida species were isolated from various clinical specimens over the study period and further speciated with antifungal susceptibility pattern. The prevalence of Candida albicans and non albicans candida are studied in relation to age, sex, site of isolation, underlying conditions and predisposing factors.

The highest numbers of candida isolates were obtained from cervical swab constituting 41(34.17\%). The other major samples were skin scrapings $18(15 \%)$, oral swabs 14(11.67\%), nail scrapings $12(10 \%)$, ear swabs $11(9.17 \%)$, sputum $10(8.33 \%)$ followed by pus $7(5.83 \%)$, urine $3(2.50 \%)$, blood 2(1.67\%) and stool $2(1.67 \%)$,According to a study done in 1980,it was found that vulvovaginitis accounted for $30 \%$ of total cases, onychia and paronychia accounted for $12 \%$,oral thrush accounted for $16 \%$ and the rest of cases were distributed among other lesions (Karabinis Andreas, et al., 1988).Amar C. Sajjan et al., 2014 also concluded the highest number of isolates was from high vaginal swab isolated from vulvovaginitis constituting 42(40.8\%). The other major samples were sputum $21(20.4 \%)$, oral swabs $12(11.7 \%)$, urine $6(5.8 \%)$, pus $6(5.8 \%)$, nail scrapings $5(4.9 \%)$, ear swabs $5(4.9 \%)$, followed by stool 4(3.9\%) and blood 1(1.67\%).Comparatively Somansu Basu et al. 2003 reported maximum 48\% candida isolates from respiratory tract specimens (Sputum, Bronchoalveolar lavage, Bronchial washing, Tracheal aspirate)followed by blood $15 \%$. In contrast, Study done by Lata R Patel et al., 2012 have reported a total of
430 Candida isolates from various clinical specimens, of which urine showed the highest number of isolates $(30.5 \%)$, followed by sputum (28.9\%) and blood $(26 \%)$. Incidence of candida species and its distribution among various clinical specimens varies between countries and between hospitals within a single country (Table 1).

In the present study C.albicans was the major species accounting for $89(74.17 \%)$ of the total isolates. Non albicans Candida constituted $21(17.5 \%)$ of C.tropicalis followed by C.glabrata, $8(6.67 \%)$ and C.krusei2 (1.67\%). Almost similar type of trend was also observed by Tavleen Jaggi et al., 2014where Candida albicans was the most frequent isolate in their study (44\%). Candida tropicalis (26.4\%), Candida parapsilosis (12.8\%), Candida glabrata (11.2\%), Candida krusei (2.4\%) and Candida guilliermondii (3.2\%) were the other common species isolated. Study done by Márcia Cristina Furlaneto et al., 2011 also showed similar results as C.albicanswas the most frequent isolate $(36 \%)$ followed by C.tropicalis (33.2\%) (Table 2).

Among the 41 cervical swabs, 28(63.63\%) isolates were C.albicans, 10 (24.39\%) were C.tropicalis, 2(4.87\%) were C.glabrata and one was C.krusei. Among 18 skin scrapings, $14(77.78 \%)$ were C.albicans, 3 (16.67\%) were C.tropicalis, $1 \quad(5.56 \%)$ was C.glabrata. Among 14 oral swabs, 10 $(71.43 \%)$ were C.albicans, $1(7.14 \%)$ was C.tropicalis, 2(14.29\%) were C.glabrata,1(7.14\%) was C.krusei. Among 12 nail scrapings, $10(83.33 \%)$ were C.albicans, $1(8.33 \%)$ each were C.tropicalis and C.glabrata. Among 11 ears swabs, $8(72.73 \%)$ were C.albicans, $2(18.18 \%)$ were C.tropicalis and 1(9.09\%) was C.glabrata. Among 10 sputum samples, 9(90\%) were C.albicans, $1(10 \%)$ was C.tropicalis, 
Among 7 pus samples, 4(57.14\%) were C.albicans, 2(28.57\%) were C.tropicalis and 1(14.29\%) was C.glabrata, Among 3 urine samples, 2(66.6\%) were C.albicans, 1(33.3\%) was C.tropicalis, Among 4 samples of blood and stool all species were C.albicans. Vinitha Mohandas et al., 2011 reported slightly different candida species distribution in various specimens, $\mathrm{C}$. albicans (39.64\%) was the most isolated species, although the Candida non albicans species with $60.36 \%$ showed the major prevalence (Table 3 ).

Age distribution of patients was from 3 months to 68 years. The highest incidence was seen in the age group 20-40 years comprising 53.3\%.Oyewole OA at el., 2013 found the highest occurrence of Candida species $(59.5 \%)$ was recorded between ages 21-30 followed by ages 31-40 with percentage occurrence of $31.0 \%$ (Table 4 ).

The Candida species were isolated more from female $(55 \%)$ than male $(45 \%)$ patients in the ratio of $1: 1.22(\mathrm{M} / \mathrm{F})$. In concordance, Amar C.S et al., 2013also found candida infection more prevalent in female patients than male with $60.2 \%$ female and $39.8 \%$ male (Table 5). Predisposing factors for candida infections differs from immune status to site of infection. Identification of risk factors is a very important way in the prevention of diseases. In the present study, pregnancy is the major predisposing factor constituting $26(21.67 \%)$ followed by HIV seropositives $25(20.83 \%)$, diabetes $19(15.83 \%)$, prolonged contact with water $14(11.67 \%)$, secondary to infections $10(8.33 \%)$ and prolonged use of drugs $8(6.67 \%)$. Others $18(15 \%)$ in predisposing factors include neutropenia, presence of malignancies, catheterization, stay in ICU, parenteral nutrition, prematurity and major surgery. A retrospective study done by SetuPatolia et al also mentioned several common predisposing factors for candida infection in medical ICU (SetuPatolia et al., 2013). NurYapar2014 evaluated the Epidemiology and risk factors for invasive candidiasis and concludes that the number of immunosuppressive patients has increased significantly in recent years. These patients are at risk for opportunistic infections, especially fungal infections. Candidiasis is one of the most frequent fungal infections determined in these immunosuppressive patients and its epidemiology has changed over the last two decades (Chun-fang Ma et al., 2013) (Table 6).

Table.1

\begin{tabular}{|l|c|c|}
\hline Clinical specimens & No. of Patients & Percentage (\%) \\
\hline Cervical swab & 41 & 34.17 \\
\hline Skin scrapings & 18 & 15.00 \\
\hline Oral swabs & 14 & 11.67 \\
\hline Nail scrapings & 12 & 10.00 \\
\hline Ear swabs & 11 & 9.17 \\
\hline Sputum & 10 & 8.33 \\
\hline Pus & 7 & 5.83 \\
\hline Urine & 3 & 2.50 \\
\hline Blood & 2 & 1.67 \\
\hline Stool & 2 & 1.67 \\
\hline
\end{tabular}


Table.2

\begin{tabular}{|l|l|l|}
\hline Species & No. of Isolates & Percentage (\%) \\
\hline C.albicans & 89 & 74.17 \\
\hline C.tropicalis & 21 & 17.5 \\
\hline C.glabrata & 8 & 6.67 \\
\hline C.krusei & 2 & 1.67 \\
\hline
\end{tabular}

Table.3 Distribution of Different Candida Species among Various Clinical Specimens

\begin{tabular}{|l|c|c|c|c|}
\hline Clinical specimens & C.albicans & C.tropicalis & C.glabrata & C.krusei \\
\hline Cervical swab & 28 & 10 & 2 & 1 \\
\hline Skin scrapings & 14 & 3 & 1 & - \\
\hline Oral swabs & 10 & 1 & 2 & 1 \\
\hline Nail scrapings & 10 & 1 & 1 & - \\
\hline Ear swabs & 8 & 2 & - & - \\
\hline Sputum & 9 & 2 & - & - \\
\hline Pus & 4 & 1 & - & - \\
\hline Urine & 2 & - & - & - \\
\hline Blood & 2 & 1 & 1 & - \\
\hline Stool & 2 & & - & - \\
\hline
\end{tabular}


Table.4

\begin{tabular}{|c|c|c|}
\hline Age & No. of Patients & Percentage (\%) \\
\hline $0-10$ & 14 & 11.67 \\
\hline $11-20$ & 10 & 8.33 \\
\hline $21-30$ & 36 & 30.00 \\
\hline $31-40$ & 28 & 23.33 \\
\hline $41-50$ & 9 & 7.50 \\
\hline $51-60$ & 11 & 9.17 \\
\hline$>61$ & 12 & 10.00 \\
\hline
\end{tabular}

Table.5

\begin{tabular}{|c|c|c|}
\hline Sex & No. of Patients & Percentage (\%) \\
\hline Male & 54 & 45 \\
\hline Female & 66 & 55 \\
\hline Total & $\mathbf{1 2 0}$ & $\mathbf{1 0 0}$ \\
\hline
\end{tabular}

Table.6

\begin{tabular}{|l|c|c|}
\hline Predisposing factors & No. of patients & Percentage (\%) \\
\hline Pregnancy & 26 & 21.67 \\
\hline HIV seropositive & 25 & 20.83 \\
\hline Diabetes & 19 & 15.83 \\
\hline Prolonged contact with water & 14 & 11.67 \\
\hline Secondary to infection & 10 & 8.33 \\
\hline $\begin{array}{l}\text { Prolonged use of corticosteroids/ } \\
\text { antibiotics }\end{array}$ & 8 & 6.67 \\
\hline Others & 18 & 15.00 \\
\hline
\end{tabular}


Table.7 Distribution of Different Species of Candida among Various Predisposing Factors

\begin{tabular}{|l|c|c|c|c|}
\hline Predisposing factors & C.albicans & C.tropicalis & C.glabrata & C.krusei \\
\hline Pregnancy & 18 & 7 & 1 & - \\
\hline HIV seropositive & 16 & 5 & 3 & 1 \\
\hline Diabetes & 15 & 3 & - & 1 \\
\hline Prolonged contact with water & 10 & 3 & 1 & - \\
\hline Secondary to other infections & 8 & 1 & 1 & - \\
\hline Prolonged use of steroid/antibiotics & 8 & - & - & - \\
\hline Others & 14 & 2 & 2 & - \\
\hline
\end{tabular}

Table.8

\begin{tabular}{|l|l|l|l|l|l|l|l|l|l|l|l|l|l|}
\hline \multirow{2}{*}{ Species } & \multicolumn{3}{|l|}{ Fluconazole } & \multicolumn{3}{|l|}{ Ketoconazole } & \multicolumn{3}{l|}{ Nystatin } \\
\cline { 2 - 15 } & S & IS & R & S & IS & R & S & IS & R & S & IS & R \\
\hline C.albicans(89) & 53 & 22 & 14 & 64 & 16 & 9 & 75 & 10 & 4 & 84 & 3 & 2 \\
\hline C.tropicalis(21) & 12 & 6 & 3 & 17 & 2 & 2 & 18 & 2 & 1 & 19 & 2 & 0 \\
\hline C.glabrata(08) & 4 & 2 & 2 & 4 & 3 & 1 & 7 & 1 & 0 & 8 & 0 & 0 \\
\hline C.krusei(02) & 1 & 1 & 0 & 2 & 0 & 0 & 2 & 0 & 0 & 2 & 0 & 0 \\
\hline Total & $\mathbf{7 0}$ & $\mathbf{3 1}$ & $\mathbf{1 9}$ & $\mathbf{8 7}$ & $\mathbf{2 1}$ & $\mathbf{1 2}$ & $\mathbf{1 0 2}$ & $\mathbf{1 3}$ & $\mathbf{5}$ & $\mathbf{1 1 3}$ & $\mathbf{5}$ & $\mathbf{2}$ \\
\hline
\end{tabular}

Among 26 Candida isolates having pregnancy as predisposing factors, 18(69.23\%) were C.albicans, 7(26.92\%) were C.tropicalis, $1 \quad(3.85 \%)$ was C.glabrata. HIV infection stood the second major predisposing factor with C.albicans as major isolates 16(64\%), followed by C.tropicalis-5(20\%), C.glabrata 3(12\%) and C.krusei 1(4 \%). C. albicans 15(78.95\%) was the major isolate in diabetes mellitus, followed by C.tropicalis $3(15.79 \%)$ and C.krusei 1(5.26\%). Among 14 Candida isolates with prolonged contact with water $10(71.43 \%)$ isolates were C.albicans, 3 $(21.43 \%)$ isolates were C.tropicalis and $1(7.14 \%)$ isolate C.glabrata. Among 10 Candida strains isolated from patients with primary bacterial infections, $8(80 \%)$ were C.albicans followed by nonalbicans Candida, C.tropicalis 1(10\%) and C.glabrata 1 (10\%). All the 8 Candida isolated from patients on prolonged antibacterial agents and corticosteroids wereC.albicans. Among the 18 isolates of 
various other predisposing factors were C.albicans 14 (77.78\%), C.tropicalis 2 $(11.11 \%)$ and C.glabrata 2(11.11\%). Although $C$. albicans is the most prevalent species involved in invasive fungal infections, the incidence of infections due to non-albicans species is increasing. Approximately $90 \%$ of infections are caused by five species: Candida albicans, Candida glabrata, Candida tropicalis, Candida parapsilosis, and Candida krusei (J. C. O. Sardi et al., 2013) (Table 7).

\section{Drug Susceptibility Pattern (Table 8)}

Monitoring antifungal resistance among Candida is useful because apart from tracking and detection of resistance, it also gives clues to emerging threats of new resistant strains. This help in assessing empirical treatment recommendation. Among 120 Candida isolates, 70(58.33\%) were sensitive to Fluconazole, 19(15.83\%) were resistant and 31(25.83\%) were intermediate sensitive. For Ketoconazole $87(72.50 \%)$ were sensitive, 12(10\%) were resistant and 21(17.50\%) were intermediate sensitive. For Nystatin 102(85\%) were sensitive, 5(4.17\%) were resistant and $13(10.83 \%)$ were intermediate sensitive. The sensitivity pattern for Amphotericin$\mathrm{B}, 113(94.17 \%)$ were sensitive, 2(1.67\%) were resistant and $5(4.17 \%)$ were intermediate sensitive.

The resistance to fluconazole was higher when compared to other drugs in the study. This might be due to widespread and indiscriminate use of fluconazole for extended periods. It is also noted that resistance was found in all the species and in different proportions. Disc diffusion method is simple,reliable and easy to perform, and could be used for preliminary screening of antifungal susceptibility testing (Chakrabarthi et al., 1995).

\section{References}

Alan, M., Sugar, Caron, A., Lyman. 1997. A practical guide to medically important fungi and the disease they cause. Lippincott-Revan, 34-58.

Amar, C., Sajjan, V.V., Mahalakshmi, Drvinay Hajare, et al. 2014. Prevalence and Antifungal Susceptibility of Candida Species Isolated From Patients Attending Tertiary Care Hospital. IOSR J. Dent. Med. Sci., (IOSR-JDMS) 13(5): 44-49.

Amar, C.S., Ashish, J., et al. 2013. Study of Prevalence and Antifungal Susceptibility of Candida. Int. J. Pharm. Bio. Sci., 4(2): 361-381.

Available online http://www.doublehelixresearch.com/D HRIJMS

Chakrabarthi, A., et al. 1995. In vitro antifungal susceptibility of Candida. Indian J. Med. Res., 102: 13-19.

ChanderJagadish. 2002. Textbook of Medical Mycology. Ed.2. New Delhi: Mehta.

Cheryl, M., Morrison, C.J. 1998. Rapid identification of Candida species with species specific DNA probes. Clin. Inf. Dis., 26: 259-74.

Chun-fang Ma, Fang-qiu Li, et al. 2013. Surveillance study of species distribution, antifungal susceptibility and mortality of nosocomial candidemia in a tertiary care hospital in China. J. Med. Microbiol., 62: 10-24.

Jarwis, W.R. 1995. Epidemiology of nosocomial fungal infections, with emphasis on Candida species. Clin. Infect. Dis., 20(6): 1526-1530.

Jawetz, et al. 1978. "Medical Mycology", Review of MedicalMicrobiology. 13th ed. Lange Medical Books/McGrowHill, Medical Publishing Division, 276-78.

Karabinis Andreas, et al. 1988. Risk factors for Candidemia in Cancer patients: A case control study. J. Clin. Microbiol., 26(3): 429-32. 
Lata, R., Patel, Jayshri, D., Pethani, Palak Bhatia, Sanjay, D., Rathod, Parul, D., Shah. 2012. Prevalence of candida infection and itsanti fungal susceptibility pattern in tertiary care hospital, Ahmedabad. Nat. J. Med. Res., 2(4): 439-441.

Manchanda, V., Agarwal, S., Verma, N. 2011. Yeast identification inroutine clinical Microbiology laboratory and its clinical relevance. Indian J. Med. Microbiol., 29(2): 172.

Márcia Cristina Furlaneto, Juliana Frasnelli Rota, et al. 2011. Species distribution and in vitro fluconazole susceptibility of clinicalCandida isolates in a Brazilian tertiary-care hospital over a 3-year period. Revista da SociedadeBrasileira de Medicina Tropical; 44(5): 595-599. doi: 10.1590/S0037-86822011000500013.

Murray, M.P., Zinchuk, R., Laronc, D.H. 2005. Chromagar candida as the sole primary medium for isolation of yeasts and as a source medium for the RapidAssimilation-of Trehalose-Test. J. Clin. Microbiol., 1210-1212.

Nur Yapar. 2014. Epidemiology and risk factors for invasive candidiasis. Therapeutics and Clinical Risk Management, 10: 95-105.

Oyewole, O.A., Okoliegbe, I.N., Alkhalil, S., Isah, P. 2013. Prevalence of Vaginal Candidiasis among Pregnant Women Attending Federal University of Technology, Minna, Nigeria, Bosso Clinic. Res. J. Pharma. Biol. Chem. Sci., 4(1): 113-120.
Rippon, J.W. 1998. Medical Mycology, Philadelphi: WB Saunders.

Sardi, J.C.O., Scorzoni, L., et al. 2013. Candida species: current epidemiology, pathogenicity, biofilm formation, natural antifungal products and new therapeutic options. J. Med. Microbiol., 62: 10-24.

Setu Patolia, Eneh Kennedy, et al. 2013. Risk factors for candida blood stream infection in medical ICU and role of colonization- A retrospective study. British J. Med. Practitioners, 6(2): a618.

Somansu Basu, Harish, C., Gugnani, Sangeeta Joshi, Neera Gupta. 2003. Distribution of Candida species indifferent clinical sources in Delhi, India and proteinase andphospholipase activity of Candida albicans isolates. Rev. Iberoam. Micol., 20: $137-140$.

Tavleen Jaggi, A.D., Urhekar, et al. 2014. Study of Candida Species in Various Clinical Samples in a Tertiary Care Hospital. DHR Int. J. Med. Sci., (DHRIJMS) 5(2): 83-88.

Vinitha Mohandas, Mamatha Ballal. 2011. Distribution of Candida Species in Different Clinical Samples and Their Virulence: Biofilm Formation, Proteinase and Phospholipase Production: A Study on Hospitalized Patients in Southern India. J. Glob. Infect. Dis., 3(1): 4-8.

\section{How to cite this article:}

Sonu Panwar and Sameer Singh Faujdar. 2016. Prevalence, Distribution, Risk factors and Antifungal Susceptibility Profiles of Candida species in a Tertiary Care Hospital. Int.J.Curr.Microbiol.App.Sci.5(4): 78-94. doi: http://dx.doi.org/10.20546/ijcmas.2016.504.039 